\title{
Botulinum Toxin Injection for Medically Refractory Neurogenic Bladder in Children: A Systematic Review
}

\author{
Shu-Yu Wu, Shang-Jen Chang, Stephen Shei-Dei Yang and Chun-Kai Hsu *(1) \\ Division of Urology, Taipei Tzu Chi Hospital, Buddhist Tzu Chi Medical Foundation, New Taipei City 23142, \\ Taiwan; nobookrain2014@gmail.com (S.-Y.W.); krissygnet@gmail.com (S.-J.C.); urolyang@gmail.com (S.S.-D.Y.) \\ * Correspondence: svevi0614@gmail.com; Tel.: +886-2-66289779 (ext. 67745); Fax: +886-2-66289009
}

check for

updates

Citation: Wu, S.-Y.; Chang, S.-J.; Yang, S.S.-D.; Hsu, C.-K. Botulinum Toxin Injection for Medically Refractory Neurogenic Bladder in Children: A Systematic Review. Toxins 2021, 13, 447. https://doi.org/ $10.3390 /$ toxins 13070447

Received: 11 May 2021

Accepted: 24 June 2021

Published: 28 June 2021

Publisher's Note: MDPI stays neutral with regard to jurisdictional claims in published maps and institutional affiliations.

Copyright: (c) 2021 by the authors. Licensee MDPI, Basel, Switzerland. This article is an open access article distributed under the terms and conditions of the Creative Commons Attribution (CC BY) license (https:// creativecommons.org/licenses/by/ $4.0 /)$.

\begin{abstract}
The objective was to evaluate the use of botulinum toxin A (BTX-A) injection in children with medically refractory neurogenic bladder. A systematic review of the literature was conducted using three databases (Medline via PubMed, Cochrane, and EMBASE). Articles evaluating BTX$\mathrm{A}$ in children with neurogenic bladder were collected. The clinical and urodynamic parameters were reviewed for the safety and efficacy evaluation. Sixteen studies were selected into this study and a total of 455 children with medical refractory neurogenic bladder were evaluated. All of the patients had received traditional conservative medications such as antimuscarinics and intermittent catheterization as previous treatment. The duration of treatments ranged from 2 months to 5.7 years. Improvements in incontinence and vesicoureteral reflux were the most common clinical outcomes. The detrusor pressure, bladder capacity and bladder compliance improvement were the most common urodynamic parameters which had been reported. However, patient satisfaction with the procedure remained controversial. There was only a minimal risk of minor adverse effects. In all of the studies, BTX-A injection was well tolerated. In conclusion, BTX-A injection appears to be a safe and effective treatment in the management of medically unresponsive neurogenic bladder in children. There is currently no evidence that the use of BTX-A injection could be used as a first-line therapy for neurogenic bladder in children.
\end{abstract}

Keywords: botulinum toxin; neurogenic bladder; children

Key Contribution: The research is related to treatment effect of onabotulinum toxin in refractory neurogenic bladder children.

\section{Introduction}

Neurogenic bladder is a complex disease which often seriously affects daily activities. It often presents as detrusor overactivity (DO), urinary leakage and hydronephrosis. DO results in decreased bladder capacity, low compliance and high intravesical pressure. These may result in cortical thinning, febrile urinary tract infection (UTI) and even loos of renal function [1,2]. Children with neurogenic bladder are at an increased risk of UTI and renal function deterioration.

Routine management includes oral medications such as anticholinergics or $\beta-3$ agonists, timely clean intermittent catheterization (CIC) or long-term indwelling catheterization $[3,4]$. The goal is to reduce intravesical pressure to protect renal function. However, if the pressure remains high or lower urinary tract symptoms persist, intravesical botulinum toxin A (BTX-A) injection is considered as alternative to irreversible surgical interventions. Current studies have demonstrated improvements in both objective symptoms and subjective parameters by intravesical BTX-A injection. BTX-A treatment may avoid or delay complex reconstructive surgery [5-8]. However, there is little evidence on the effects of BTX-A on pediatric neurogenic bladder patients. Therefore, the aim of this study was to perform a systematic review of intravesical BTX-A injection on treating children with refractory neurogenic voiding dysfunction. 


\section{Materials and Methods}

We used the Preferred Reporting Items for Systematic Reviews and Meta-Analyses (PRISMA) guidelines to report this systematic review [9]. We did not register a protocol for this systematic review. Three databases (Medline via PubMed, Cochrane, and EMBASE) were searched for qualified studies. The following keywords were used, along with Boolean operators: "neurogenic", "bladder", and "botulinum toxin". The following search strategy was used on Medline via PubMed: (Botox, "neurogenic bladder") and (botulinum toxin, "neurogenic bladder"). The search was conducted on 1 February 2021. Only articles published in English which included patients aged under 18 years were collected.

After removing duplicates, the titles and abstracts of the studies were reviewed by two independent reviewers ( $\mathrm{Hsu}$ and $\mathrm{Wu}$ ). If the article is selected only by one reviewer, it will be retained first and analyzed in the next step. Case reports, current review articles, commentaries, editorials, letters and abstracts were excluded. Studies focusing on adult patients and those that included children along with others but did not report the outcomes of each subgroup separately were also excluded. Then the full texts were obtained and reviewed by all the authors. For any other related research, references were also checked manually. Studies focusing on non-neurogenic bladder were excluded. For each article, the following parameters were collected: type of study; patient numbers and genders; mean age of the patients; neurological disease(s); BTX-A treatment modality and duration; and clinical and urodynamic parameters for the safety and efficacy evaluation.

\section{Results}

A total of 30 studies were screened, of which 16 were included according to the PRISMA protocols (Figure A1). Of these 16 studies, five were prospective without placebo control. Only two randomized control trials were identified. A total of 455 patients with neurogenic bladder were evaluated, ranging from seven to 65 patients per study. The most common neurologic deficit was meningomyelocele. The patients with a poor response to clean intermittent catheterization or medical treatment were treated with BTX-A injections into the detrusor of urinary bladder or the external sphincter. The age of the patients varied widely in these patients. The dosage of BTX-A ranged from $100 \mathrm{U}$ to $1200 \mathrm{U}$, most studies use the onabotulinum toxin (100-500 IU, 10-50 IU / $\mathrm{kg}$ according to the body weight); only two studies use the onabobotulinum toxin (500-1200 IU, $40 \mathrm{U} / \mathrm{kg}$ according to the body weight). The number of injection sites range from 20 to 50. Ten studies showed the results of multiple injections. Four studies applied reinjection after the urodynamics parameters had returned to baseline or in cases of persistent or recurrent incontinence. All of the studies used an interval of at least 3 months between each injection, with the interval ranging from months to years. There was a trend of a stable urodynamic response after multiple injections. All of the studies reported a low adverse event rate and that the injection was safe. The clinical demographic data are shown in Table 1. 
Table 1. Summary of studies evaluating the use of botulinum toxin A in children with neurogenic bladder (patients, treatment, and response characteristics).

\begin{tabular}{|c|c|c|c|c|c|c|c|c|c|c|c|c|}
\hline First Author & Type of Study & $\begin{array}{l}\text { Number of } \\
\text { Patients } \\
(n, \mathrm{M} / \mathrm{F})\end{array}$ & $\begin{array}{c}\text { Mean Age } \\
\text { (Year) }\end{array}$ & $\begin{array}{l}\text { Neurologic } \\
\text { Diseases }(n)\end{array}$ & $\begin{array}{c}\text { Previous } \\
\text { Treatment } \\
\text { Modalities }\end{array}$ & $\begin{array}{l}\text { Treatment } \\
\text { Modality }\end{array}$ & $\begin{array}{l}\text { Treatment } \\
\text { Duration }\end{array}$ & $\begin{array}{l}\text { Follow Up } \\
\text { Period }\end{array}$ & $\begin{array}{c}\text { Clinical } \\
\text { Parameters }\end{array}$ & $\begin{array}{l}\text { Urodynamic } \\
\text { Parameters }\end{array}$ & $\begin{array}{l}\text { Adverse } \\
\text { Event }(n)\end{array}$ & $\begin{array}{l}\text { Primary and } \\
\text { Special } \\
\text { Outcomes }\end{array}$ \\
\hline $\begin{array}{l}\text { Heinrich } \\
\text { Schulte- } \\
\text { Baukloh } \\
{[8]}\end{array}$ & $\begin{array}{l}\text { Retrospective } \\
\text { chart analysis }\end{array}$ & $10,6 / 4$ & 11.2 & $\begin{array}{c}\text { MMC 8 } \\
\text { Intraspinal } \\
\text { astrocytoma 1 } \\
\text { SCI 1 }\end{array}$ & $\begin{array}{c}\text { anticholinergic } \\
\text { medications } \\
\text { and CIC }\end{array}$ & $\begin{array}{l}12 \mathrm{IU} / \mathrm{kg} \text {, diluted } \\
\text { in } 15 \text { to } 20 \mathrm{~mL} \\
\text { normal saline, } \\
\text { injected into } 30 \text { to } \\
50 \text { sites }\end{array}$ & $\begin{array}{l}\text { repeated } \\
\text { injection (at } \\
\text { least three } \\
\text { injections; } 4 \\
\text { had received } \\
\text { five or more } \\
\text { injections) }\end{array}$ & $\begin{array}{c}1,3 \text {, and } 6 \\
\text { months }\end{array}$ & NR & $\begin{array}{l}\text { Reflex volume, } \\
\text { Pdetmax, } \\
\text { maximun } \\
\text { bladder } \\
\text { capacity, } \\
\text { bladder } \\
\text { compliance }\end{array}$ & NR & $\begin{array}{c}\text { After the first } \\
\text { versus the fifth } \\
\text { injection } \\
\text { reflex volume } \\
\text { increased by } 81 \% \\
\text { versus } 88 \% \text {, } \\
\text { maximal } \\
\text { detrusor pressure } \\
\text { decreased by } 7 \% \\
\text { versus } 39 \% \\
\text { maximal } \\
\text { cystometric } \\
\text { bladder capacity } \\
\text { increased by } 88 \% \\
\text { versus } 72 \% \\
73 \% \text { complete }\end{array}$ \\
\hline $\begin{array}{c}\text { Abdol- } \\
\text { Mohammad } \\
\text { Kajbafzadeh } \\
\text { [10] }\end{array}$ & $\begin{array}{c}\text { Single-center, } \\
\text { prospective, } \\
\text { unrandomized } \\
\text { study }\end{array}$ & $26,20 / 6$ & 6.9 & MMC & $\begin{array}{c}\text { anticholinergic } \\
\text { medications } \\
\text { and CIC }\end{array}$ & $\begin{array}{l}10 \mathrm{IU} / \mathrm{kg} \text {, diluted } \\
\text { in } 20 \mathrm{~mL} \text { of } \\
\text { normal saline, } \\
\text { injected } \\
\text { intravesically } \\
\text { into at least } 40 \\
\text { sites }\end{array}$ & single injection & 4 months & $\begin{array}{l}\text { Incontinence, } \\
\text { VUR, urinary } \\
\text { tract infection, } \\
\text { fecal } \\
\text { continence }\end{array}$ & $\begin{array}{c}\text { Pdetmax, } \\
\text { maximun } \\
\text { bladder } \\
\text { capacity }\end{array}$ & $\begin{array}{c}\text { no major } \\
\text { treatment } \\
\text { related event }\end{array}$ & $\begin{array}{l}\text { dry, } 88 \% \text { total } \\
\text { improvement in } \\
\text { urine } \\
\text { incontinence; } \\
\text { significant } \\
\text { improvements in } \\
\text { mean maximal } \\
\text { detrusor pressure } \\
\text { and average } \\
\text { maximal bladder } \\
\text { capacity }\end{array}$ \\
\hline $\begin{array}{l}\text { W. Altaweel } \\
\text { [11] }\end{array}$ & $\begin{array}{l}\text { Prospective } \\
\text { study }\end{array}$ & $20,8 / 12$ & 13 & MMC & $\begin{array}{c}\text { anticholinergic } \\
\text { medications } \\
\text { and CIC }\end{array}$ & $\begin{array}{c}5 \mathrm{IU} / \mathrm{kg} \\
\text { (maximum } 300 \\
\text { IU) diluted } 10 \\
\text { times in normal } \\
\text { saline, injected to } \\
\text { give } 10 \mathrm{IU} \text { per } \\
\text { site }\end{array}$ & $\begin{array}{c}\begin{array}{c}\text { repeated } \\
\text { injection } \\
\text { (reassess }\end{array} \\
\text { approximately } \\
3 \text { months after } \\
\text { each treatment, } \\
\text { repeat injection } \\
\text { if incontinent) }\end{array}$ & $\begin{array}{c}3 \text { months after } \\
\text { each treatment } \\
\text { (total mean } \\
\text { follow-up 17.2 } \\
\text { months) }\end{array}$ & $\begin{array}{l}\text { Incontinence, } \\
\text { hydronephro- } \\
\text { sis }\end{array}$ & $\begin{array}{l}\text { Pdetmax, } \\
\text { bladder } \\
\text { compliance }\end{array}$ & $\begin{array}{l}\text { no treatment } \\
\text { related event }\end{array}$ & $\begin{array}{c}65 \% \text { continent; } \\
\text { significant } \\
\text { improvements in } \\
\text { mean bladder } \\
\text { capacity, } \\
\text { maximum } \\
\text { detrusor pressure } \\
\text { and compliance }\end{array}$ \\
\hline
\end{tabular}


Table 1. Cont.

\begin{tabular}{|c|c|c|c|c|c|c|c|c|c|c|c|c|}
\hline First Author & Type of Study & $\begin{array}{l}\text { Number of } \\
\text { Patients } \\
(n, M / F)\end{array}$ & $\begin{array}{c}\text { Mean Age } \\
\text { (Year) }\end{array}$ & $\begin{array}{l}\text { Neurologic } \\
\text { Diseases }(n)\end{array}$ & $\begin{array}{c}\text { Previous } \\
\text { Treatment } \\
\text { Modalities }\end{array}$ & $\begin{array}{l}\text { Treatment } \\
\text { Modality }\end{array}$ & $\begin{array}{l}\text { Treatment } \\
\text { Duration }\end{array}$ & $\begin{array}{l}\text { Follow Up } \\
\text { Period }\end{array}$ & $\begin{array}{c}\text { Clinical } \\
\text { Parameters }\end{array}$ & $\begin{array}{l}\text { Urodynamic } \\
\text { Parameters }\end{array}$ & $\begin{array}{l}\text { Adverse } \\
\text { Event }(n)\end{array}$ & $\begin{array}{l}\text { Primary and } \\
\text { Special } \\
\text { Outcomes }\end{array}$ \\
\hline $\begin{array}{l}\text { Saeed Safari } \\
\text { [12] }\end{array}$ & $\begin{array}{l}\text { Prospective, } \\
\text { single center, } \\
\text { double-blind, } \\
\text { randomized } \\
\text { control trial }\end{array}$ & $\begin{array}{l}\text { Group A (30, } \\
\text { 13/17) } \\
\text { Group B (30, } \\
\text { 14/16) }\end{array}$ & $\begin{array}{c}\text { Group A } \\
(6.58) \\
\text { Group B } \\
(6.71)\end{array}$ & MMC & $\begin{array}{l}\text { anticholinergic } \\
\text { medications } \\
\text { and CIC }\end{array}$ & $\begin{array}{c}\text { Group A (10 } \\
\mathrm{U} / \mathrm{kg} \text {, diluted in } \\
20 \mathrm{~mL} \text { of normal } \\
\text { saline, injected } \\
\text { into } 40 \text { points of } \\
\text { bladder); } \\
\text { Group B ( } \mathrm{U} / \mathrm{kg} \text {, } \\
\text { injected in the } \\
\text { same way as in } \\
\text { group A and the } \\
\text { remaining (2 } \\
\mathrm{U} / \mathrm{kg}) \text { was } \\
\text { injected by } 4 \\
\text { additional } \\
\text { injections in } \\
\text { external urethral } \\
\text { sphincter) } \\
10\end{array}$ & single injection & 3 and 6 months & $\begin{array}{l}\text { Incontinence } \\
\text { episodes, } \\
\text { Constipation, } \\
\text { Vesicoureteral } \\
\text { reflux grade }\end{array}$ & $\begin{array}{l}\text { Detrusor- } \\
\text { sphincter } \\
\text { dyssinergia, } \\
\text { maximum } \\
\text { detrusor } \\
\text { pressure, } \\
\text { post-voiding } \\
\text { residual volumn, } \\
\text { bladder capacity }\end{array}$ & NR & $\begin{array}{l}\text { BTX-A injections } \\
\text { in both sphincter } \\
\text { and detrusor } \\
\text { seems to have } \\
\text { extra benefits in } \\
\text { voiding }\end{array}$ \\
\hline $\begin{array}{l}\text { Aniruddh V } \\
\text { Deshpande } \\
\text { [13] }\end{array}$ & $\begin{array}{l}\text { Prospective, } \\
\text { non- } \\
\text { randomized } \\
\text { study }\end{array}$ & $7,6 / 1$ & 16 & Spinal bifida & $\begin{array}{c}\text { CIC and } \\
\text { oxybutynin } \\
\text { (dose range } 5 \\
\text { mg b.d.-5 mg } \\
\text { q.i.d.) }\end{array}$ & $\begin{array}{l}\text { (maximum } 300 \\
\text { IU) diluted as } 10 \\
\text { units per } \mathrm{mL} \text { in } \\
\text { saline, injected } \\
\text { into the detrusor } \\
\text { at approximately } \\
20 \text { to } 30 \text { sites }\end{array}$ & single injection & $\begin{array}{c}1,3-6 \text { and } 9 \\
\text { months }\end{array}$ & $\begin{array}{l}\text { Incontinence, } \\
\text { satisfaction } \\
\text { score }\end{array}$ & $\begin{array}{l}\text { Bladder capacity, } \\
\text { bladder } \\
\text { compliance }\end{array}$ & UTI 1 & $\begin{array}{l}\text { significant } \\
\text { improvements in } \\
\text { bladder } \\
\text { compliance and } \\
\text { incontinence }\end{array}$ \\
\hline R Le Nué [14] & $\begin{array}{l}\text { Retrospective } \\
\text { chart analysis }\end{array}$ & $8,3 / 5$ & 12.4 & $\begin{array}{l}\text { SCI } 6 \\
\text { Stroke } 2\end{array}$ & $\begin{array}{l}\text { maximal oral } \\
\text { anticholinergic } \\
\text { treatment, CIC }\end{array}$ & $\begin{array}{c}12 \mathrm{IU} / \mathrm{kg} \\
\text { (maximal } 300 \mathrm{IU} \text { ), } \\
\text { diluting to a } \\
\text { concentration of } \\
100 \mathrm{IU} / 10 \mathrm{~mL} \\
\text { before } 2008 \mathrm{and} \\
\text { then } 100 \mathrm{IU} / 5 \\
\mathrm{~mL} \text { of } 0.9 \% \\
\text { saline, injected } \\
\text { into } 10 \text { to } 30 \text { sites }\end{array}$ & $\begin{array}{l}\text { repeated } \\
\text { injection (2-6 } \\
\text { injections, } \\
\text { repeat } \\
\text { injections } \\
\text { depended on } \\
\text { the initial } \\
\text { urodynamic } \\
\text { status) }\end{array}$ & $\begin{array}{c}6 \text { months after } \\
\text { each treatment } \\
\text { (total mean } \\
\text { follow-up } 47 \\
\text { months) }\end{array}$ & $\begin{array}{l}\text { continence } \\
\text { score }\end{array}$ & $\begin{array}{c}\text { Pdetmax, } \\
\text { bladder } \\
\text { compliance, } \\
\text { maximun } \\
\text { bladder capacity, } \\
\text { safe capacity }\end{array}$ & NR & $\begin{array}{l}\text { Improvements in } \\
\text { the mentioned } \\
\text { parameters }\end{array}$ \\
\hline
\end{tabular}


Table 1. Cont

\begin{tabular}{|c|c|c|c|c|c|c|c|c|c|c|c|c|}
\hline First Author & Type of Study & $\begin{array}{l}\text { Number of } \\
\text { Patients } \\
(n, M / F)\end{array}$ & $\begin{array}{c}\text { Mean Age } \\
\text { (Year) }\end{array}$ & $\begin{array}{l}\text { Neurologic } \\
\text { Diseases }(n)\end{array}$ & $\begin{array}{c}\text { Previous } \\
\text { Treatment } \\
\text { Modalities }\end{array}$ & $\begin{array}{l}\text { Treatment } \\
\text { Modality }\end{array}$ & $\begin{array}{l}\text { Treatment } \\
\text { Duration }\end{array}$ & $\begin{array}{l}\text { Follow Up } \\
\text { Period }\end{array}$ & $\begin{array}{c}\text { Clinical } \\
\text { Parameters }\end{array}$ & $\begin{array}{l}\text { Urodynamic } \\
\text { Parameters }\end{array}$ & $\begin{array}{l}\text { Adverse } \\
\text { Event }(n)\end{array}$ & $\begin{array}{l}\text { Primary and } \\
\text { Special } \\
\text { Outcomes }\end{array}$ \\
\hline $\begin{array}{c}\text { Maya Horst } \\
\text { [15] }\end{array}$ & $\begin{array}{l}\text { Retrospective } \\
\text { chart analysis }\end{array}$ & $11,1 / 10$ & 6.7 & MMC & $\begin{array}{c}\text { anticholinergic } \\
\text { medications }\end{array}$ & $\begin{array}{c}10 \mathrm{IU} / \mathrm{kg} \\
\text { (maximal } 300 \mathrm{IU}), \\
\text { diluting to a } \\
\text { concentration of } \\
100 \mathrm{IU} / 10 \mathrm{~mL} \text { of } \\
0.9 \% \text { saline, each } \\
\text { injection } \\
\text { contained } 0.3-0.5 \\
\mathrm{~mL}\end{array}$ & $\begin{array}{c}\text { repeated } \\
\text { injection (1-4 } \\
\text { injections, } \\
\text { reinjection was } \\
\text { performed if } \\
\text { the } \\
\text { urodynamic } \\
\text { follow up } \\
\text { study showed } \\
\text { compliance } \\
\text { and pressure } \\
\text { returned to } \\
\text { baseline } \\
\text { values) }\end{array}$ & $\begin{array}{c}3 \text { and } 12 \\
\text { months after } \\
\text { each injection }\end{array}$ & VUR & $\begin{array}{c}\text { Changes in } \\
\text { bladder } \\
\text { compliance, } \\
\text { maximal bladder } \\
\text { capacity, } \\
\text { maximal } \\
\text { detrusor pressure }\end{array}$ & NR & $\begin{array}{c}\text { detrusor pressure } \\
\text { decreased by } 17 \% \\
\text { and bladder } \\
\text { capacity } \\
\text { increased by } 33 \% \text {; } \\
\text { similar effect on } \\
\text { capacity and } \\
\text { detrusor pressure } \\
\text { could be } \\
\text { achieved with } \\
\text { repeated } \\
\text { injection }\end{array}$ \\
\hline $\begin{array}{c}\text { Pawel Kroll } \\
\text { [16] }\end{array}$ & $\begin{array}{l}\text { prospective, } \\
\text { non-placebo- } \\
\text { controlled } \\
\text { study }\end{array}$ & $65,34 / 31$ & 6.7 & $\begin{array}{c}\text { MMC } 61 \\
\text { Sacral agenesis } 3 \\
\text { Cerebral palsy } 1\end{array}$ & $\begin{array}{l}\text { CIC and oral } \\
\text { oxybutynine }\end{array}$ & $\begin{array}{c}50 \mathrm{IU} / \mathrm{kg} \\
\text { (maximal } 500 \mathrm{IU} \text { ), } \\
\text { diluted in } 10 \mathrm{~mL} \\
\text { normal saline, } \\
\text { injected each } \\
\text { with } 0.5 \mathrm{~mL} \text { of } \\
\text { the solution }\end{array}$ & single injection & $\begin{array}{l}6 \text { and } 12 \\
\text { months }\end{array}$ & continence & $\begin{array}{l}\text { Maximun } \\
\text { catheterized } \\
\text { volume, } \\
\text { maximun } \\
\text { volume of leak } \\
\text { point pressure }\end{array}$ & NR & $\begin{array}{l}\text { Improvements in } \\
\text { the mentioned } \\
\text { parameters }\end{array}$ \\
\hline $\begin{array}{c}\text { Antonio Marte } \\
\text { [17] }\end{array}$ & $\begin{array}{l}\text { Retrospective } \\
\text { chart analysis }\end{array}$ & $47,25 / 22$ & 10.7 & MMC & $\begin{array}{l}\text { anticholinergic } \\
\text { medications } \\
\text { and CIC }\end{array}$ & $\begin{array}{l}200 \mathrm{IU} \text {, diluted in } \\
20 \mathrm{~mL} \text { of } 0.9 \% \\
\text { saline solution, } \\
\text { not exceeding the } \\
\text { dosage of } 12 \\
\mathrm{IU} / \mathrm{kg} \text {, injected } \\
\text { for a total of } 20 \\
\text { injections }\end{array}$ & $\begin{array}{c}\text { repeated } \\
\text { injection (1-3 } \\
\text { injections, for } \\
\text { the recurrence } \\
\text { of symptoms) }\end{array}$ & $\begin{array}{c}\text { 6, } 12 \text { and } 24 \\
\text { weeks after } \\
\text { each injection } \\
\text { (total mean } \\
\text { followup } 5.7 \\
\text { years) }\end{array}$ & $\begin{array}{l}\text { Incontinence, } \\
\text { VUR }\end{array}$ & $\begin{array}{l}\text { Mean leak point } \\
\text { volume, mean } \\
\text { leak point } \\
\text { pressure, specific } \\
\text { capacity at } 20 \mathrm{~cm} \\
\mathrm{H}_{2} \mathrm{O} \text {, bladder } \\
\text { capacity }\end{array}$ & $\begin{array}{c}\text { slight } \\
\text { hematuria } \\
38 \text {, UTI 2, } \\
\text { gastric pain } \\
\text { 2, facial } \\
\text { flushing 2, } \\
\text { mild } \\
\text { hyposthenia } \\
\text { of the lower } \\
\text { limbs 5 }\end{array}$ & $\begin{array}{c}\text { significant } \\
66.45 \% \text { average } \\
\text { increase of leak } \\
\text { point volume, } \\
\text { significant } \\
118.57 \% \text { average } \\
\text { increase of } \\
\text { specific bladder } \\
\text { capacity at } 20 \mathrm{~cm} \\
\mathrm{H}_{2} \mathrm{O}\end{array}$ \\
\hline V. Figueroa [5] & $\begin{array}{l}\text { Prospective } \\
\text { study }\end{array}$ & 17 & 10.7 & $\begin{array}{c}\text { spina } \\
\text { bifida/spinal } \\
\text { dysraphism }\end{array}$ & $\begin{array}{l}\text { anticholinergic } \\
\text { medications }\end{array}$ & $\begin{array}{c}10 \mathrm{IU} / \mathrm{kg} \\
\text { (maximal } 200 \mathrm{IU} \text { ), } \\
\text { diluted in normal } \\
\text { saline } \\
\text { to a } \\
\text { concentration of } \\
10 \text { units/cc }\end{array}$ & $\begin{array}{l}\text { repeated } \\
\text { injection } \\
\text { (average of } 2.5 \\
\text { injec- } \\
\text { tions/patient; } \\
\text { range, 1-6) }\end{array}$ & $\begin{array}{c}3,6 \text { months } \\
\text { after each } \\
\text { injection (total } \\
\text { mean } \\
\text { follow-up } 4 \\
\text { years) }\end{array}$ & Incontinence & $\begin{array}{c}\text { Mean bladder } \\
\text { capacity, detrusor } \\
\text { compliance, } \\
\text { bladder volume, } \\
\text { bladder } \\
\text { compliance }\end{array}$ & NR & $\begin{array}{l}\text { The optimal } \\
\text { response occurs } \\
\text { with a maximum } \\
\text { administration of } \\
\text { BTX-A up to } 300 \\
\text { units. }\end{array}$ \\
\hline
\end{tabular}


Table 1. Cont

\begin{tabular}{|c|c|c|c|c|c|c|c|c|c|c|c|c|}
\hline First Author & Type of Study & $\begin{array}{l}\text { Number of } \\
\text { Patients } \\
(n, M / F)\end{array}$ & $\begin{array}{l}\text { Mean Age } \\
\text { (Year) }\end{array}$ & $\begin{array}{l}\text { Neurologic } \\
\text { Diseases }(n)\end{array}$ & $\begin{array}{c}\text { Previous } \\
\text { Treatment } \\
\text { Modalities }\end{array}$ & $\begin{array}{l}\text { Treatment } \\
\text { Modality }\end{array}$ & $\begin{array}{l}\text { Treatment } \\
\text { Duration }\end{array}$ & $\begin{array}{l}\text { Follow Up } \\
\text { Period }\end{array}$ & $\begin{array}{c}\text { Clinical } \\
\text { Parameters }\end{array}$ & $\begin{array}{l}\text { Urodynamic } \\
\text { Parameters }\end{array}$ & $\begin{array}{l}\text { Adverse } \\
\text { Event }(n)\end{array}$ & $\begin{array}{l}\text { Primary and } \\
\text { Special } \\
\text { Outcomes }\end{array}$ \\
\hline $\begin{array}{c}\text { Sang Woon } \\
\text { Kim [18] }\end{array}$ & $\begin{array}{l}\text { Retrospective } \\
\text { chart analysis }\end{array}$ & $37,22 / 15$ & 7.49 & $\begin{array}{c}\text { Spina bifida } 29 \\
\text { Syrinx } 1 \\
\text { Cerebral palsy } 4 \\
\text { Guillain-Barre } \\
\text { syndrome } 1 \\
\text { Spinal cord } \\
\text { hemangioma } 1 \\
\text { Post meningitis } \\
\text { sequelae 1 }\end{array}$ & $\begin{array}{l}\text { high-dose } \\
\text { anticholinergic } \\
\text { medications }\end{array}$ & $\begin{array}{c}10 \mathrm{IU} / \mathrm{kg} \\
\text { (maximal } 200 \mathrm{IU} \text { ), } \\
\text { diluting to a } \\
\text { concentration of } \\
100 \mathrm{IU} / 5 \mathrm{~mL} \text { of } \\
0.9 \% \text { saline, } \\
\text { injected into } 20 \text { to } \\
40 \text { sites }\end{array}$ & single injection & $1,3,6$ months & $\begin{array}{c}\text { Patient Global } \\
\text { Impression of } \\
\text { Improvement } \\
\text { (PGI-I) }\end{array}$ & $\begin{array}{c}\text { detrusor- } \\
\text { sphincter } \\
\text { dyssynergia, } \\
\text { maximum } \\
\text { cystometric } \\
\text { capacity, } \\
\text { estimated } \\
\text { bladder capacity, } \\
\text { Residual urine } \\
\text { volume, maximal } \\
\text { detrusor } \\
\text { pressure, reflex } \\
\text { detrusor volume, } \\
\text { bladder } \\
\text { compliance, open } \\
\text { bladder neck }\end{array}$ & UTI 1 & $\begin{array}{l}\text { preoperative } \\
\text { bladder } \\
\text { compliance and } \\
\text { open bladder } \\
\text { neck were } \\
\text { important } \\
\text { predictors }\end{array}$ \\
\hline $\begin{array}{l}\text { M. K. Khan } \\
\text { [19] }\end{array}$ & $\begin{array}{l}\text { Retrospective } \\
\text { chart analysis }\end{array}$ & $22,16 / 6$ & 10 & $\begin{array}{c}\text { Myelomeningocele } \\
10 \\
\text { Anorectal } \\
\text { malformation } 3 \\
\text { Spinal cord } \\
\text { trauma } 3 \\
\text { Tethered cord } \\
\text { syndrome 2 } \\
\text { Caudal } \\
\text { regression } \\
\text { syndrome 2 } \\
\text { Sacrococcygeal } \\
\text { teratoma 1 } \\
\text { Transverse } \\
\text { myelitis 1 }\end{array}$ & $\begin{array}{l}\text { anticholinergic } \\
\text { medications }\end{array}$ & $\begin{array}{c}10 \mathrm{IU} / \mathrm{kg} \\
\text { (maximal } 300 \mathrm{IU} \text { ), } \\
\text { diluted in normal } \\
\text { saline } \\
\text { to a } \\
\text { concentration of } \\
10 \text { units/cc }\end{array}$ & $\begin{array}{c}\text { repeated } \\
\text { injection (Four } \\
\text { patients have } \\
\text { received two } \\
\text { or more } \\
\text { injections for } \\
\text { the recurrence } \\
\text { of symptoms) }\end{array}$ & $\begin{array}{c}\text { 3, } 6 \text { months } \\
\text { after each } \\
\text { injection (total } \\
\text { mean } \\
\text { follow-up } 11 \\
\text { months, range } \\
\text { 3-38) }\end{array}$ & $\begin{array}{l}\text { Incontinence, } \\
\text { hydronephro- } \\
\text { sis }\end{array}$ & $\begin{array}{c}\text { Cystometric } \\
\text { bladder capacity, } \\
\text { mean maximum } \\
\text { detrusor pressure }\end{array}$ & NR & $\begin{array}{c}\text { patients with } \\
\text { anticholinergics } \\
\text { intolerance seen } \\
\text { to have be more } \\
\text { effective after } \\
\text { BTX-A injection } \\
\text { than those with } \\
\text { anticholinergic } \\
\text { refractory }\end{array}$ \\
\hline $\begin{array}{l}\text { Cagri Akin } \\
\text { Sekerci [20] }\end{array}$ & $\begin{array}{l}\text { Retrospective } \\
\text { chart analysis }\end{array}$ & $19,4 / 15$ & 10.3 & myelodysplasia & $\begin{array}{l}\text { anticholinergic } \\
\text { medications }\end{array}$ & $\begin{array}{c}10 \mathrm{IU} / \mathrm{kg} \\
\text { (maximal } 200 \mathrm{IU} \text { ), } \\
\text { diluted in normal } \\
\text { saline } \\
\text { to a } \\
\text { concentration of } \\
10 \text { units/cc, } \\
\text { injected to give } \\
10 \mathrm{IU} \text { per site }\end{array}$ & $\begin{array}{c}\text { repeated } \\
\text { injection (1-5 } \\
\text { injections, } \\
\text { repeated only } \\
\text { after the } \\
\text { relapse of } \\
\text { incontinence } \\
\text { accompanying } \\
\text { deterioration } \\
\text { in urodynamic } \\
\text { findings) }\end{array}$ & $\begin{array}{c}\text { every } 3 \\
\text { months after } \\
\text { each injection }\end{array}$ & $\begin{array}{l}\text { Incontinence, } \\
\text { VUR, hy- } \\
\text { dronephrosis }\end{array}$ & $\begin{array}{l}\text { Maximum } \\
\text { cystometric } \\
\text { capacity, } \\
\text { Maximum } \\
\text { detrusor } \\
\text { pressure, } \\
\text { Compliance }\end{array}$ & hematuria 2 & $\begin{array}{l}\text { significant } \\
\text { improvements in } \\
\text { the mentinal } \\
\text { parameters after } \\
\text { repeat injection }\end{array}$ \\
\hline
\end{tabular}


Table 1. Cont.

\begin{tabular}{|c|c|c|c|c|c|c|c|c|c|c|c|c|}
\hline First Author & Type of Study & $\begin{array}{l}\text { Number of } \\
\text { Patients } \\
(n, \mathrm{M} / \mathrm{F})\end{array}$ & $\begin{array}{c}\text { Mean Age } \\
\text { (Year) }\end{array}$ & $\begin{array}{l}\text { Neurologic } \\
\text { Diseases }(n)\end{array}$ & $\begin{array}{c}\text { Previous } \\
\text { Treatment } \\
\text { Modalities }\end{array}$ & $\begin{array}{l}\text { Treatment } \\
\text { Modality }\end{array}$ & $\begin{array}{l}\text { Treatment } \\
\text { Duration }\end{array}$ & $\begin{array}{l}\text { Follow Up } \\
\text { Period }\end{array}$ & $\begin{array}{c}\text { Clinical } \\
\text { Parameters }\end{array}$ & $\begin{array}{l}\text { Urodynamic } \\
\text { Parameters }\end{array}$ & $\begin{array}{l}\text { Adverse } \\
\text { Event }(n)\end{array}$ & $\begin{array}{l}\text { Primary and } \\
\text { Special } \\
\text { Outcomes }\end{array}$ \\
\hline $\begin{array}{c}\text { Juliette } \\
\text { Hascoet [21] }\end{array}$ & $\begin{array}{l}\text { Retrospective } \\
\text { chart analysis }\end{array}$ & $53,28 / 25$ & 8.5 & Spina bifida & $\begin{array}{c}\text { anticholinergic } \\
\text { medications }\end{array}$ & $100-500 \mathrm{U}$ & $\begin{array}{c}\text { repeated } \\
\text { injection (1-8 } \\
\text { injections) }\end{array}$ & $\begin{array}{l}\text { mean follow } \\
\text { up of } 3.7 \text { years }\end{array}$ & Incontinence & $\begin{array}{c}\text { Resolution of } \\
\text { detrusor } \\
\text { overactivity, } \\
\text { normal bladder } \\
\text { compliance, } \\
\text { maximum, } \\
\text { cystometric } \\
\text { capacity, } \\
\text { maximum } \\
\text { detrusor pressure }\end{array}$ & $\begin{array}{c}3 \text { UTI } \\
\text { episodes out } \\
\text { of } 141 \\
\text { injections } \\
(2.1 \%)\end{array}$ & $\begin{array}{c}66 \% \text { clinical } \\
\text { success rate, } 34 \% \\
\text { urodynamic } \\
\text { success rate }\end{array}$ \\
\hline $\begin{array}{l}\text { Shehryer } \\
\text { Naqvi [22] }\end{array}$ & $\begin{array}{l}\text { Retrospective } \\
\text { chart analysis }\end{array}$ & $30,15 / 15$ & 7.4 & $\begin{array}{c}\text { MMC 18, } \\
\text { Lipomeningo- } \\
\text { coele 4, } \\
\text { Transverse } \\
\text { myelitis 1, Sacral } \\
\text { agenesis } 3\end{array}$ & $\begin{array}{l}\text { anticholinergic } \\
\text { medications } \\
\text { and CIC }\end{array}$ & $\begin{array}{l}40 \mathrm{IU} / \mathrm{kg} \text {, } \\
\text { (maximal } 1200 \\
\mathrm{IU} \text { ) diluted in } 20 \\
\text { mL sodium } \\
\text { chloride solution }\end{array}$ & $\begin{array}{c}\text { repeated } \\
\text { injection } \\
\text { (Median } \\
\text { number of } \\
\text { injections was } \\
3 \text { (range } 2 \text { to } 5 \text { ) }\end{array}$ & $\begin{array}{c}\text { median } 2 \\
\text { months (range } \\
0-29)\end{array}$ & $\begin{array}{c}\text { incontinence, } \\
\text { VUR }\end{array}$ & $\begin{array}{c}\text { Bladder } \\
\text { compliance, } \\
\text { cystometric } \\
\text { capacity, } \\
\text { maximum } \\
\text { neurogenic } \\
\text { detrusor } \\
\text { overactivity }\end{array}$ & $\begin{array}{l}\text { abdominal } \\
\text { pain 1, UTI } 1\end{array}$ & $\begin{array}{l}\text { significantly } \\
\text { improved } \\
\text { cystometric } \\
\text { capacity and } \\
\text { maximum } \\
\text { neurogenic } \\
\text { detrusor } \\
\text { overactivity, no } \\
\text { significant } \\
\text { difference in } \\
\text { urodynamic } \\
\text { parameters } \\
\text { between first and } \\
\text { last injections }\end{array}$ \\
\hline $\begin{array}{l}\text { Khalid Fouda } \\
\text { Neel [23] }\end{array}$ & $\begin{array}{c}\text { Prospective, } \\
\text { single center, } \\
\text { randomized } \\
\text { trial }\end{array}$ & $\begin{array}{c}\text { Group 1: } 12 \\
\text { 9/3 Group 2: } \\
116 / 7\end{array}$ & $\begin{array}{l}\text { Group 1: } \\
\text { 6.1, Group } \\
\text { 2: } 5.1\end{array}$ & MMC & $\begin{array}{l}\text { oxybutynin } \\
\text { and CIC }\end{array}$ & $\begin{array}{c}12 \mathrm{IU} / \mathrm{kg} \\
\text { (maximal } 300 \mathrm{IU}) \\
\text { Group 1: } \\
\text { continued to } \\
\text { receive } \\
\text { anticholinergics; } \\
\text { Group 2: } \\
\text { dicontinued to } \\
\text { receive } \\
\text { anticholinergics }\end{array}$ & single injection & 12 months & incontinence & $\begin{array}{l}\text { Maximum } \\
\text { cystometric } \\
\text { capacity, } \\
\text { maximum } \\
\text { detrusor pressure }\end{array}$ & NR & $\begin{array}{l}\text { Oxybutynin did } \\
\text { not have } \\
\text { augmentative } \\
\text { effect after } \\
\text { BTX-A injection }\end{array}$ \\
\hline
\end{tabular}

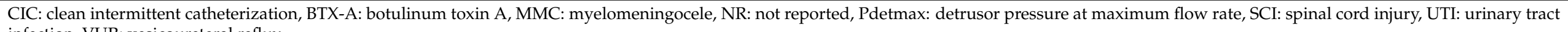
infection, VUR: vesicoureteral reflux. 


\subsection{Clinical Parameters}

Among the 16 studies, 15 of them report the improvement of clinical parameters. The clinical parameters included incontinence, vesicoureteral reflux (VUR), UTI, bowel dysfunction, hydronephrosis and patient reported satisfaction. The most commonly reported symptom was urinary incontinence, and 13 studies reported an improvement in incontinence, with a post-injection improvement rate of 54-100\%. Moreover, 30-100\% of the patients were totally continent after the injection. The second most commonly reported symptom was VUR, and six studies reported improvements in VUR. Of these studies, only one reported no change in VUR after the injection [15]. The other studies reported improving or improved reflux, with a rate of improvement ranging from $73-100 \%$. Other clinical parameters including hydronephrosis, fecal continence and UTI were rarely reported. Three studies reported an improvement in hydronephrosis $[11,19,20]$.

In these studies, some of the patients had improvements in hydronephrosis but others did not, although the number of patients was limited (3-10 patients in each study). The improvements in hydronephrosis were reported to be related to resolving reflux. Bowel dysfunction was only reported in two studies [10,12], and the improvement rate ranged from $46-93 \%$. One study reported improvements in UTI [10], in which 26 children with a previous UTI did not have an episode during four months of follow-up after BTX-A injection. The patient's reported response was also an important clinical parameter. Two studies reported opposite results of patient satisfaction and changes in urodynamics $[13,18]$. The only study that mentioned urethral injections was a prospective, randomized, doubleblind control trial by Safari et al. They reported that BTX-A injections in both the sphincter and detrusor seemed to be more beneficial in decreasing postvoiding residual volume and reducing symptoms compared with an intradetrusor injection alone even unable to facilitate spontaneous emptying [12].

\subsection{Urodynamic Parameters}

Urodynamic changes were also reported in the studies. Although the items emphasized in each article were different, they mainly focused on detrusor pressure, bladder capacity and bladder compliance. Ten studies reported baseline urodynamic DO according to urodynamic studies before the injection. Eleven studies reported a significant decrease in maximum detrusor pressure (Pdet). The patients in these studies had a very high Pdet at baseline (43-139 $\mathrm{cm} \mathrm{H} \mathrm{H}_{2} \mathrm{O}$ ), which then decreased to $22-83 \mathrm{~cm} \mathrm{H}_{2} \mathrm{O}$ after the injection. In addition, bladder pressure was reported to be reduced by up to $50 \%$. Fourteen studies reported enlarged bladder capacity after BTX-A injection, ranging from 1.45 to 3.64 times (40-217 mL) compared to baseline. Bladder compliance was also significantly improved in these studies. Among the seven prospective studies, bladder compliance was reported in three $[5,11,13]$. Deshpande et al. reported that the effect on bladder compliance was most pronounced 3 months after the injection. They reported that bladder compliance improved from $4.42 \mathrm{~mL} / \mathrm{cm} \mathrm{H}_{2} \mathrm{O}$ at baseline, to $9.33 \mathrm{~mL} / \mathrm{cm} \mathrm{H}_{2} \mathrm{O}$ at 3 months, and then the effect progressively decreased $\left(8.12\right.$ and $6.35 \mathrm{~mL} / \mathrm{cm} \mathrm{H}_{2} \mathrm{O}$ at 6 and 9 months after the injection, respectively) [13]. Altaweel et al. reported significant improvements in bladder compliance after both the first injection (from 5.2 to $13 \mathrm{~mL} / \mathrm{cm} \mathrm{H}_{2} \mathrm{O}$ ) and second injection (from 6 to $15.1 \mathrm{~mL} / \mathrm{cm} \mathrm{H}_{2} \mathrm{O}$ ) [11]. Figueroa et al. [5]. reported the effect of repeat injections. Compared with baseline, after the first injection, the mean bladder compliance improved by $45.2 \%$; after subsequent injections, the value increased by $55.1 \%$. In Neel et al.'s prospective randomized control trial, continuous oxybutynin treatment after BTX-A injection did not provide extra benefits based on the urodynamic parameters (Pdet, bladder capacity) [23] In addition to the general parameters mentioned above, Marte's study focused on leak point volume (LPV), leak point pressure (LPP), and the specific volume at $20 \mathrm{~cm} \mathrm{H}_{2} \mathrm{O}$ pressure (SC 20) [17]. They reported a significant average increase in LPV $(66.45 \%)$ and in SC $20(118.57 \%)$ after surgery. However, the difference between preoperative and postoperative LPP was not significant. Two studies also investigated the reflex volume $[9,18]$ and reported improvements similar to bladder capacity, although the statistical difference 
remained controversial. In the 11 studies which reported the effect of repeat injections, there were two important findings. First, the effect of BTX-A decreased with time, usually after 6-9 months. However, repeat injections after symptom relapse were still as beneficial as the first injection. Second, in the patients in whom the first BTX-A injection was not as effective as expected, repeat injections provided better results.

\subsection{Safety and Side Effects}

BTX-A injections appeared to be safe, as only a few patients had adverse events. Across all retrieved studies, six studies mentioned adverse effects of BTX-A injections. The most common adverse effect was temporary hematuria, and one study reported a high rate $(80.9 \%)$ of post-injection hematuria [17]. However, another study showed a much lower rate of hematuria (10.9\%) [20]. Post-injection UTI were the other reported adverse effects. Five studies reported the rates of UTI after each injection $(14.3 \%, 4.3 \%, 2.7 \%, 2.1 \%$ and $3.3 \%$ respectively) $[13,17,18,21,22]$. The other studies reported mild or even no side effects.

\section{Discussion}

Neurogenic bladder presents with various bladder dysfunctions, including detrusorsphincter dyssynergia, DO and poor compliance. Failure of control will lead to recurrent UTI and upper urinary tract deterioration and loss of renal function. Urinary symptoms are present in nearly all of these patients, in whom renal failure and UTI may cause death [24,25]. Preservation of renal function and elimination of incontinence are crucial in bladder management while improving quality of life.

Oral antimuscarinics often act as first line therapy for detrusor overactivity or poor compliance. Many antimuscarinics are currently available worldwide. However, oxybutynin is the only agent approved by the FDA on children. The effectiveness of treatment is well known as well as its adverse event including dry eyes, dry skin, constipation and stomach-intestine disorders. Moreover, it can also cause disturbances require dose adjustment or even discontinuation of oxybutynin treatment. Recently, a new beta-3 agonist agent has become available as add-on therapy or alternative. If pharmacological treatments fail, surgery such as bladder augmentation will be carried out as final solution. However, those complex surgical procedures yield long-term serious complications such as stenosis, stone formation, infection or metabolic disturbance [26,27].

BTX-A is a 2-chain peptide neurotoxin protein produced by the Gram-positive, anaerobic bacterium Clostridium botulinum. Physiologic activation starts by proteolytically break down into the $100 \mathrm{kDa}$ heavy chain and $50 \mathrm{kDa}$ light chain. The light chain of the botulinum neurotoxin binds with high specificity to the glycoprotein on cholinergic nerve terminals. Ultimately, BTX-A causes acetylcholine-containing vesicles unable to reach the nerve terminal. This blockage results in muscle paralysis and relax of urinary bladder [3,28]

Application of BTX-A in neurourologic field has increased in past two decades. Schurch et al. first reported efficacy of intradetrusor injections of BTX-A in 2000 [25]. After a series of trials, BTX-A injection have become the standard of care for neurogenic detrusor overactivity refractory to antimuscarinics in adults with spinal cord injury or multiple sclerosis. However, in pediatric neurogenic bladder, BTX-A is applied as substitute to anticholinergics when adverse events such as urinary incontinence or upper urinary tract dilation persisted. If BTX-A does not work, reconstructive surgery of the lower urinary tract is final resort to preserve renal function [29]. In addition, BTX-A injections could be used as a diagnostic tool for the evaluation of neurogenic incontinence caused by bladder outlet incompetence.

In the studies, intravesical BTX-A injections improved both clinical symptoms and cystometric parameters in children who got neurogenic detrusor overactivity (NDO) [5-7,10]. The first report of the use of BTX-A injection in children was published by Schulte-Baukloh in 2002 [9]. Subsequently, several studies have reported that BTA injections can improve both urodynamic parameters (reflex volume, bladder capacity, maximal detrusor pressure and bladder compliance) and symptoms of NDO $[5,9,28,30]$. The clinical outcomes were 
favorable with a completely resolved urinary incontinence in one third to nearly $100 \%$ of the patients. In addition, there was high percentage of clinical incontinence resolution, although the degree of improvement varies between each study. Post-injection UTI were the most common adverse events. Other significant adverse events which are commonly noted in adults such as increasing post-void residual urine, acute urine retention, and general muscle weakness were rarely mentioned.

The International Children's Continence Society (ICCS) does not define which urodynamic outcome parameters should be evaluated. Therefore, bladder compliance, maximum detrusor pressure (MDP) and maximum cystometric capacity were most commonly used for outcome analysis. An increase in maximum cystometric capacity and decrease in MDP were reported in nearly all of the studies. However, in some series, the MDP was still above $40 \mathrm{~cm}-\mathrm{H} 2 \mathrm{O}$ after BTX-A injection. The increase in bladder compliance was also statistically significant. Bladder augmentation or other reconstructive surgery accompany with perioperative risk and long-term adverse event. To avoid or at least delay major operation is the ultimate goal of oral pharmacologic treatment and intradetrusor injection in pediatric neurogenic patients. Most of the enrolled studies did not report the number of patients who end up with bladder augmentation despite BTX-A injections. Furthermore, the studies did not report in how many major reconstructive surgery was delayed during follow-up, and it was not clear if these patients all had a low-compliance bladder. Still, effort on avoiding irreversible major operation is meaningful for pediatric patients because of complications and possible cancer development decades after augmentation. Interesting, the most important renal function deterioration was not mentioned in all literatures. We have many studies focus on the improvement of VUR and hydronephrosis, but not the exact renal function by the blood test or other examinations. It might due to the difficulty for children to draw blood and the lack of long-term research.

Although forming neutralizing antibodies may downgrade treatment effect after repeated injection of BTX-A. The problem has seldom been reported before, and the levels of antibodies did not increase after repeat injections [31]. BTX-A injections may lead to axonal sprouting and the generation of new synaptic contacts on paralyzed muscle fibers. The problem was reported in striated muscle rather than smooth muscle of urinary bladder. No structural effects have been shown on the effects of BTX-A injections on the human detrusor. Recurrent puncture during repeated injections of BTX-A may also result in scarring and subsequent reduction of bladder compliance [32]. Despite the concerns, repeated BTX-A injection still showed similar improvement of urodynamic parameters in each intervention. The lasting effect varies from 8 to 15 months in enrolled studies.

Besides traditional intradetrusor BTX-A injections, there are also other methods of delivery such as electromotive drug application (EMDA) and liposomal drug delivery. EMDA uses iontophoresis and electrophoresis to deliver the pharmacological agents into deeper tissue layers. Two electrodes will be used to provide electric current during the operation. EMDA has been used widely in delivering different medication into human bladder wall in adult urology [33]. Kajbafzadeh et al. have published two studies about BTX-A delivery use EMDA in 2011 [34,35]. Through immunohistochemical staining, they showed that EMDA provided more BTX-A penetration in bladder suburothelial layers compare to the traditional injection. In their trial, EMDA got significant improvement in maximum cystometric capacity, mean reflex volume and mean end-fill pressure. In a more recent study in 2019, Koh et al. reported that BTX-A/EMDA got poor performance when compared with conventional intravesical BTX-A injections. The negative finding may imply that large size of the BTX-A molecule is hard to penetrate the thick and hypertrophy bladder wall in NDO [36]. Liposomes are another theoretically ideal carrier in transurothelial drug delivery. It could penetrate the urothelial barrier through endocytosis. The recent clinical studies showed that BTX-A encapsulated within liposomes (Lipo-BTX) may be useful in adult patients with a non-neurogenic overactive bladder [37-39]. It provided benefit on frequency and urgency but not on urinary incontinence or residual urine volume. Therefore, Lipo-BTX could affect the afferent signaling of the urothelium 
but not work on the efferent motor activity of the detrusor. Although Lipo-BTX may be effective for the storage symptoms of an overactive bladder, the application on children with refractory neurogenic bladder remain uncertain due to minimal effects on decreasing detrusor contraction.

BTX-A injection on neurogenic bladder children result in good outcomes in clinical or urodynamic parameters in most of the studies. Although antimuscarinics was applied as first line treatment, threat of no response and/or side effects persisted. BTX-A injection was proposed to be a favorable second-line intervention after antimuscarinics in refractory neurogenic bladder. The safety of BTX-A injection has previously been reported in the literature $[17,18,21,22]$. In these studies, the rate of adverse events ranged from $2-5 \%$. Constipation, fatigue, headache, and hypertension are most common adverse events. After all, BTX-A injection is favorable safe in all of the included studies.

\section{Conclusions}

BTX-A injections for refractory neurogenic bladder are effective in resolving both urinary incontinence and improving urodynamic parameters in most children. To maintain the effect, multiple injections are often needed. General anesthesia is routinely carried out in children for BTX-A injections. Alternative route has been investigated. However, there is no definite result in pediatric setting. From enrolled studies, BTX-A may injection is a favorable intervention in avoidance of major reconstructive surgery such as bladder augmentation. Our results provide further evidence that intradetrusor BTX-A injection is a safe and effective option in the management of children with neurogenic bladder who fail to respond to conservative medical therapy.

Author Contributions: Conceptualization, S.-J.C. and S.S.-D.Y.; methodology, S.-J.C.; software, C.K.H.; validation, S.-Y.W. and C.-K.H.; formal analysis, C.-K.H.; investigation, C.-K.H.; resources, S.S.-D.Y.; data curation, C.-K.H.; writing-original draft preparation, S.-Y.W.; writing—review and editing, C.-K.H.; visualization, C.-K.H.; supervision, S.-J.C.; project administration, S.S.-D.Y.; funding acquisition, S.S.-D.Y. All authors have read and agreed to the published version of the manuscript.

Funding: This research received no specific grant from any funding agency in the public, commercial, or not-for-profit sectors.

Institutional Review Board Statement: Not applicable.

Informed Consent Statement: Not applicable.

Data Availability Statement: Data available in a publicly accessible repository.

Acknowledgments: We would like to show our gratitude to Hann-Chorng Kuo for sharing his pearls of wisdom with us during the course of this research. We thank all the reviewers for their valuable comments and suggestions, which helped us to improve the quality of the article.

Conflicts of Interest: The authors declare no conflict of interest. 


\section{Appendix A}

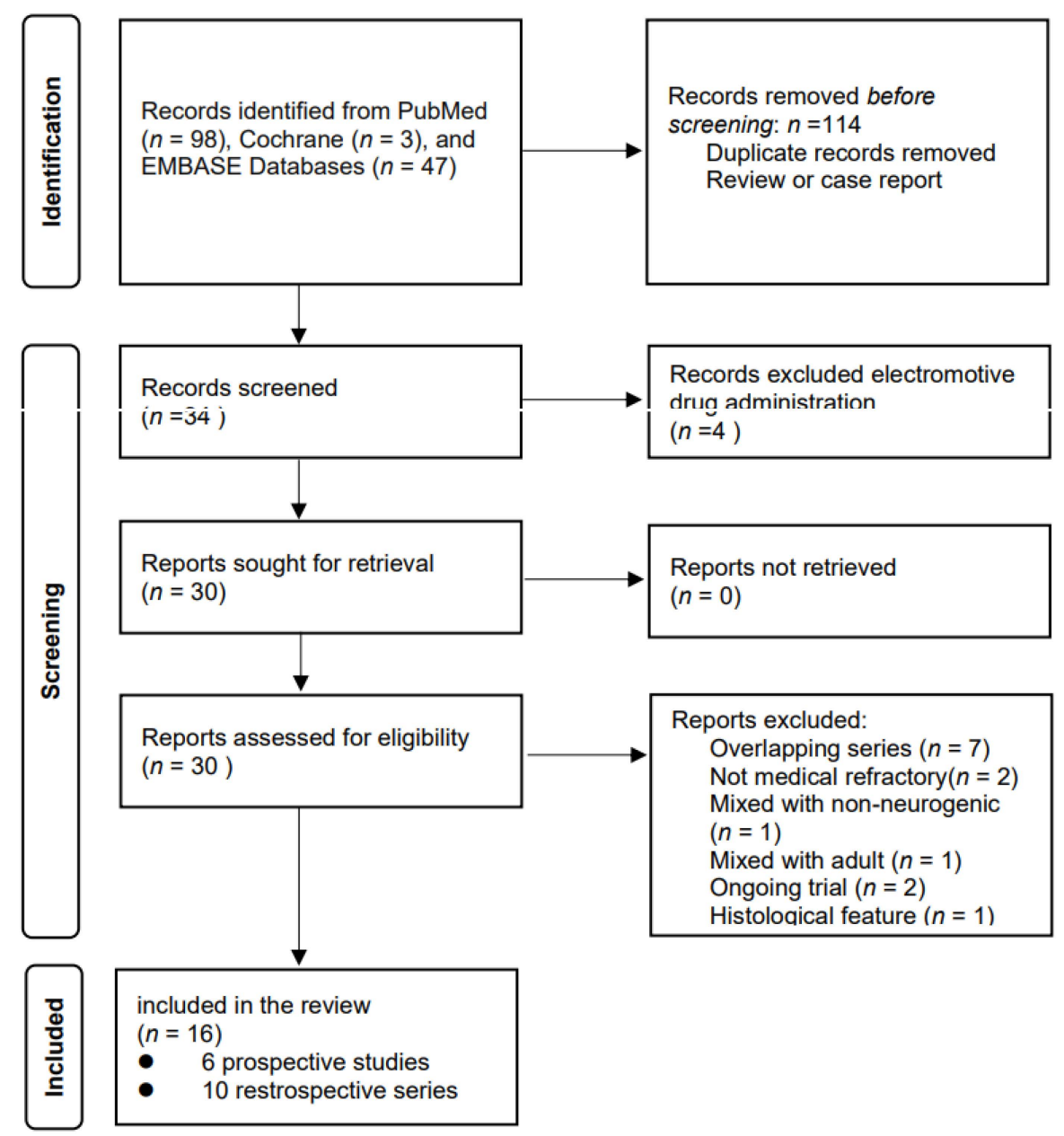

Figure A1. Flowchart detailing the search strategy and selection of the included studies.

\section{References}

1. Dik, P.; Klijn, A.J.; van Gool, J.D.; de Jong-de Vos van Steenwijk, C.C.E.; de Jong, T.P.V.M. Early start to therapy preserves kidney function in spina bifida patients. Eur. Urol. 2006, 49, 908-913. [CrossRef]

2. Kasabian, N.G.; Bauer, S.B.; Dyro, F.M.; Colodny, A.H.; Mandell, J.; Retik, A.B. The prophylactic value of clean intermittent catheterization and anticholinergic medication in newborns and infants with myelodysplasia at risk of developing urinary tract deterioration. Am. J. Dis. Child. 1992, 146, 840-843. [CrossRef]

3. Nitti, V.W. Botulinum toxin for the treatment of idiopathic and neurogenic overactive bladder: State of the art. Rev. Urol. 2006, 8, 198-208.

4. Lee, B.; Featherstone, N.; Nagappan, P.; McCarthy, L.; O’Toole, S. British Association of Paediatric Urologists consensus statement on the management of the neuropathic bladder. J. Pediatr. Urol. 2016, 12, 76-87. [CrossRef]

5. Figueroa, V.; Romao, R.; Salle, J.L.P.; Koyle, M.A.; Braga, L.H.P.; Bägli, D.J.; Lorenzo, A.J. Single-center experience with botulinum toxin endoscopic detrusor injection for the treatment of congenital neuropathic bladder in children: Effect of dose adjustment, multiple injections, and avoidance of reconstructive procedures. J. Pediatr. Urol. 2014, 10, 368-373. [CrossRef]

6. Riccabona, M.; Koen, M.; Schindler, M.; Goedele, B.; Pycha, A.; Lusuardi, L.; Bauer, S.B. Botulinum-A toxin injection into the detrusor: A safe alternative in the treatment of children with myelomeningocele with detrusor hyperreflexia. J. Urol. 2004, 171, 845-848. [CrossRef]

7. Akbar, M.; Abel, R.; Seyler, T.M.; Bedke, J.; Haferkamp, A.; Gerner, H.J.; Möhring, K. Repeated botulinum-A toxin injections in the treatment of myelodysplastic children and patients with spinal cord injuries with neurogenic bladder dysfunction. BJU Int. 2007, 100, 639-645. [CrossRef]

8. Schulte-Baukloh, H.; Michael, T.; Schobert, J.; Stolze, T.; Knispel, H.H. Efficacy of botulinum-A toxin in children with detrusor hyperreflexia due to myelomeningocele: Preliminary results. Urology 2002, 59, 325-327. [CrossRef] 
9. Moher, D.; Shamseer, L.; Clarke, M.; Ghersi, D.; Liberati, A.; Petticrew, M.; Shekelle, P.; Stewart, L.A.; PRISMA-P Group. Preferred reporting items for systematic review and meta-analysis protocols (PRISMA-P) 2015 statement. Syst. Rev. 2015, 4, 1. [CrossRef]

10. Kajbafzadeh, A.M.; Moosavi, S.; Tajik, P.; Arshadi, H.; Payabvash, S.; Salmasi, A.H.; Akbari, H.R.; Nejat, F. Intravesical injection of botulinum toxin type A: Management of neuropathic bladder and bowel dysfunction in children with myelomeningocele. Urology 2006, 68, 1091-1096. [CrossRef]

11. Altaweel, W.; Jednack, R.; Bilodeau, C.; Corcos, J. Repeated intradetrusor botulinum toxin type A in children with neurogenic bladder due to myelomeningocele. J. Urol. 2006, 175, 1102-1105. [CrossRef]

12. Safari, S.; Jamali, S.; Habibollahi, P.; Arshadi, H.; Nejat, F.; Kajbafzadeh, A.M. Intravesical injections of botulinum toxin type A for management of neuropathic bladder: A comparison of two methods. Urology 2010, 76, 225-230. [CrossRef] [PubMed]

13. Deshpande, A.V.; Sampang, R.; Smith, G.H.H. Study of botulinum toxin A in neurogenic bladder due to spina bifida in children. ANZ J. Surg. 2010, 80, 250-253. [CrossRef] [PubMed]

14. Nué, R.L.; Harper, L.; Sèze, M.D.; Bouteiller, C.; Goossens, D.; Dobremez, E. Evolution of the management of acquired neurogenic bladder in children using intradetrusor botulinum toxin type A injections: 5-year experience and perspectives. J. Pediatr. Urol. 2012, 8, 497-503. [CrossRef]

15. Horst, M.; Weber, D.M.; Bodmer, C.; Gobet, R. Repeated Botulinum-A toxin injection in the treatment of neuropathic bladder dysfunction and poor bladder compliance in children with myelomeningocele. Neurourol. Urodyn. 2011, 30, 1546-1549. [CrossRef] [PubMed]

16. Kroll, P.; Jankowski, A.; Soltysiak, J.; Murias, M.; Skrzypczak, M.; Zachwieja, J. Botulinum toxin-A injections in children with neurogenic bladder. Nephro Urol Mon. 2011, 3, 125-128.

17. Marte, A. Onabotulinumtoxin A for Treating Overactive/Poor Compliant Bladders in Children and Adolescents with Neurogenic Bladder Secondary to Myelomeningocele. Toxins 2013, 5, 16-24. [CrossRef]

18. Kim, S.W.; Choi, J.H.; Lee, Y.S.; Han, S.W.; Im, Y.J. Preoperative urodynamic factors predicting outcome of botulinum toxin-A intradetrusor injection in children with neurogenic detrusor overactivity. Urology 2014, 84, 1480-1484. [CrossRef]

19. Khan, M.K.; Brink, B.A.V.; DeFoor, W.R.; Minevich, E.; Jackson, E.; Noh, P.; Reddy, P.P. Botulinum toxin injection in the pediatric population with medically refractory neuropathic bladder. J. Pediatr. Urol. 2016, 12, 104.e1-104.e6. [CrossRef]

20. Sekerci, C.A.; Tanidir, Y.; Garayev, A.; Akbal, C.; Tarcan, T.; Simsek, F. Clinical and Urodynamic Results of Repeated IntradetrusorOnabotulinum Toxin A Injections in Refractory Neurogenic Detrusor Overactivity: Up to 5 Injections in a Cohort of Children With Myelodysplasia. Urology 2018, 111, 168-175. [CrossRef]

21. Hascoet, J.; Peyronnet, B.; Forin, V.; Baron, M.; Capon, G.; Prudhomme, T.; Allenet, C.; Tournier, S.; Maurin, C.; Cornu, J.N.; et al. Intra-Detrusor Injections of Botulinum Toxin Type a in Children with Spina Bifida: A Multicenter Study. Urology 2018, 116, 161-167. [CrossRef] [PubMed]

22. Naqvi, S.; Clothier, J.; Wright, A.; Garriboli, M. Urodynamic Outcomes in Children after Single and Multiple Injections for Overactive and Low Compliance Neurogenic Bladder Treated with Abobotulinum Toxin A. J. Urol. 2020, 203, 413-419. [CrossRef]

23. Neel, K.F.; Soliman, S.; Salem, M.; Seida, M.; Al-Hazmi, H.; Khatab, A. Botulinum-A toxin: Solo treatment for neuropathic noncompliant bladder. J. Urol. 2007, 178, 2593-2597. [CrossRef]

24. Veenboer, P.W.; Bosch, J.L.H.R.; van Asbeck, F.W.A.; de Kort, L.M.O. Upper and lowerurinary tract outcomes in adult myelomeningocele patients: A systematic review. PLoS ONE 2012, 7, e48399. [CrossRef]

25. Schurch, B.; Stöhrer, M.; Kramer, G.; Schmid, D.M.; Gaul, G.; Hauri, D. Botulinum-Atoxin for treating detrusor hyperreflexia in spinal cord injured patients: A new alternative to anticholinergic drugs? Preliminary results. J. Urol. 2000, 164, 692-697. [CrossRef]

26. Alloussi, S.; Mürtz, G.; Braun, R.; Gerhardt, U.; Heinrich, M.; Hellmis, E.; Horn, W.; Marschall-Kehrel, D.; Niklas, K.; Raabe, M. Efficacy, tolerability and safety of propiverine hydrochloride in comparison to oxybutynin in children with urge incontinence due to overactive bladder: Results of a multicentre observational cohort study. BJU Int. 2010, 106, 550-556. [CrossRef]

27. Uçar, M.; Akgül, A.K.; Parlak, A.; Yücel, C.; Kılıç, N.; Balkan, E. Non-invasive evaluation of botulinum-A toxin treatment efficacy in children with refractory overactive bladder. Int. Urol. Nephrol. 2018, 50, 1367-1373. [CrossRef]

28. Frevert, J. Pharmaceutical, biological, and clinical propertiesof botulinum neurotoxin type A products. Drugs R D 2015, 15, 1-9. [CrossRef]

29. Scheepe, J.R.; Blok, B.F.M.; Hoen, L.A. Applicability of botulinum toxin type A in paediatric neurogenic bladder management. Curr. Opin. Urol. 2017, 27, 14-19. [CrossRef]

30. Mangera, A.; Apostolidis, A.; Andersson, K.E.; Dasgupta, P.; Giannantoni, A.; Roehrborn, C.; Novara, G.; Chapple, C. An updated systematic review and statistical comparison of standardised mean outcomes for the use of botulinum toxin in the management of lower urinary tract disorders. Eur. Urol. 2014, 65, 981-990. [CrossRef]

31. Schulte-Baukloh, H.; Herholz, J.; Bigalke, H.; Miller, K.; Knispel, H.H. Results of a BoNT/A antibody study in children and adolescents after onabotulinumtoxin A (Botox ${ }^{\circledR}$ ) detrusor injection. Urol. Int. 2011, 87, 434-438. [CrossRef] [PubMed]

32. Haferkamp, A.; Schurch, B.; Reitz, A.; Krengel, U.; Grosse, J.; Kramer, G.; Schumacher, S.; Bastian, P.J.; Büttner, R.; Müller, S.C. Lack of ultrastructural detrusor changes following endoscopic injection of botulinum toxin type a in overactive neurogenic bladder. Eur. Urol. 2004, 46, 784-791. [CrossRef] [PubMed]

33. Giannantoni, A.; Di Stasi, S.M.; Chancellor, M.B.; Costantini, E.; Porena, M. New frontiers in intravesical ltherapies and drug delivery. Eur. Urol. 2006, 50, 1183-1193. [CrossRef] [PubMed] 
34. Kajbafzadeh, A.M.; Montaser-Kouhsari, L.; Ahmadi, H.; Sotoudeh, M. Intravesical electromotive botulinum toxin type A administration: Part I—experimental study. Urology 2011, 77, 1460-1464. [CrossRef] [PubMed]

35. Kajbafzadeh, A.M.; Ahmadi, H.; Montaser-Kouhsari, L.; Sharifi-Rad, L.; Nejat, F.; Bazargan-Hejazi, S. Intravesical electromotive botulinum toxin type A administration-Part II: Clinical application. Urology 2011, 77, 439-445. [CrossRef] [PubMed]

36. Koh, C.; Melling, C.V.; Jennings, C.; Lewis, M.; Goyal, A. Efficacy of electromotive drug administration in delivering botulinum toxin a in children with neuropathic detrusor overactivity-outcomes of a pilot study. J. Pediatr. Urol. 2019, 15, 552.e1-552.e8. [CrossRef] [PubMed]

37. Chang, H.I.; Yeh, M.K. Clinical development of liposome-based drugs: Formulation, characterization, and therapeutic efficacy. Int. J. Nanomed. 2012, 7, 49-60.

38. Rajaganapathy, B.R.; Chancellor, M.B.; Nirmal, J.; Dang, L.; Tyagi, P. Bladder uptake of liposomes after intravesical administration occurs by endocytosis. PLoS ONE 2015, 10, e0122766. [CrossRef]

39. Chuang, Y.C.; Kaufmann, J.H.; Chancellor, D.D.; Chancellor, M.B.; Kuo, H.C. Bladder instillation of liposome encapsulated onabotulinumtoxin A improves overactive bladder symptoms: A prospective, multicenter, double-blind, randomized trial. J. Urol. 2014, 192, 1743-1749. [CrossRef] 